\title{
Control Design for Discrete-Time Fuzzy Systems with Disturbance Inputs via Delta Operator Approach
}

\author{
Qi Zhou, ${ }^{1}$ Yabin Gao, ${ }^{1}$ Hongyi Li, $^{2}$ and Hamid Reza Karimi ${ }^{3}$ \\ ${ }^{1}$ College of Information Science and Technology, Bohai University, Jinzhou, Liaoning 121013, China \\ ${ }^{2}$ College of Engineering, Bohai University, Jinzhou, Liaoning 121013, China \\ ${ }^{3}$ Department of Engineering of the Faculty of Engineering and Science, University of Agder, Grimstad, Norway
}

Correspondence should be addressed to Hongyi Li; lihongyi2009@gmail.com

Received 21 August 2013; Accepted 22 September 2013

Academic Editor: Tao Li

Copyright (C) 2013 Qi Zhou et al. This is an open access article distributed under the Creative Commons Attribution License, which permits unrestricted use, distribution, and reproduction in any medium, provided the original work is properly cited.

\begin{abstract}
This paper is concerned with the problem of passive control design for discrete-time Takagi-Sugeno (T-S) fuzzy systems with time delay and disturbance input via delta operator approach. The discrete-time passive performance index is established in this paper for the control design problem. By constructing a new type of Lyapunov-Krasovskii function (LKF) in delta domain, and utilizing some fuzzy weighing matrices, a new passive performance condition is proposed for the system under consideration. Based on the condition, a state-feedback passive controller is designed to guarantee that the resulting closed-loop system is very-strictly passive. The existence conditions of the controller can be expressed by linear matrix inequalities (LMIs). Finally, a numerical example is provided to demonstrate the feasibility and effectiveness of the proposed method.
\end{abstract}

\section{Introduction}

It is well known that the fuzzy logic control [1-4] is one of the most effective approaches to handle complex nonlinear systems. Takagi-Sugeno (T-S) fuzzy model has been proposed in [5] and applied to formulate a complex nonlinear systems into a framework. Some affine local models can be interpolated by a set of fuzzy membership functions in this framework. By T-S fuzzy model, a set of complex nonlinear systems can be possibly described as a weighed sum of some simple linear subsystems. Recently, the problem of stability analysis and controller synthesis of nonlinear systems in T-S fuzzy model has been extensively investigated in [5-18]. Due to the effect of time delay in systems, the problems of T-S fuzzy systems with time delays have got considerable attention in recent years, and some results have been developed in $[9,19-31]$

Recently, due to the fact that the passive properties can keep the system internally stable and have been frequently used to improve the stability of control systems. The passivity control has been widely applied in various engineering areas, such as electrical circuits systems, complex networks systems, mechanical systems, and nonlinear systems. The problems of passivity analysis and passive control for systems have been widely investigated [32-38]. The passive control problem has been investigated for fuzzy systems $[35,39,40]$. Among them, the authors in [40] are concerned with the very strict passive controller design problem for T-S fuzzy systems with timevarying delay.

It is well known that the best system performances can be obtained with the shorter sampling period. In [41, 42], the authors pointed out that the excellent finite word length performance can be achieved under fast sampling via delta operator approach. The authors in [43] introduced the transformations between shift operator and delta operator transfer function models. A technique was developed in [44] to obtain an approximate delta operator system for a given continuous system. In [45], a tabular method was presented for real polynomial root distribution with respect to a circle in the complex plane, which is useful in stability of delta operator 
formulated discrete-time systems with a sampling time. By using delta operator formulation, the main design features of a loop transfer recovery controller both at input and output have been overviewed in [46]. More recently, many stability analysis and controller synthesis results about delta operator approach have been proposed in [47-49]. To mention a few, the robust stabilization problem was investigated in [47] for delta operator systems with time-varying delays. The authors in [49] investigated the robust $H_{\infty}$ control problem for a class of T-S fuzzy systems with time delays by using delta operator approach. However, there have been few results on the passive control for T-S fuzzy systems with time delays and disturbance inputs via delta operator approach, which motivates this study.

In this paper, the problem of passive control is investigated for discrete-time T-S fuzzy systems with time delay and disturbance input via delta operator approach. The discrete-time passive performance index is concerned in this paper for the control design problem. A novel type Lyapunov-Krasovskii functional (LKF) is constructed in delta domain to present a new passive performance condition for discrete-time T-S fuzzy systems. Based on the condition, a state-feedback passive controller is designed to guarantee that the resulting closed-loop system is very strictly passive. The controller existence condition can be obtained in terms of linear matrix inequalities (LMIs), which can be solved by the standard software. A numerical example is given to illustrate the effectiveness of the proposed approach.

At first, the T-S fuzzy model is employed to represent the nonlinear systems. By applying the LMIs techniques and LKF in $\delta$-domain, the problem of passive control design for the discrete T-S fuzzy system with time delay and disturbance inputs is chewed. Then a new fuzzy state-feedback controller is designed which guarantees that the closed-loop fuzzy delta operator system with time delay is robustly asymptotically stable and satisfies a prescribed passive performance level. And those are some key points of contribution. It is worthwhile to note that a faster sampling method is utilized, and hence a better control effect by applying delta operator approach than shift operator approach is achieved. Finally, a numerical example is shown to indicate the feasibility and effectiveness of the proposed method.

This paper is organized as follows. The problem to be solved is formulated in Section 2. Main results, including passive analysis and passive controller design, are presented in Section 3. Section 4 provides an illustrative example to show the effectiveness and potential of the proposed design techniques. It is concluded this paper in Section 5.

Notation. The notation used throughout the paper is fairly standard. $L_{2}[0, \infty)$ denotes the space of square-integrable vector functions over $[0, \infty)$. The notation $X>0$ (resp., $X \geq$ 0 ), for $X \in \mathbb{R}^{n \times n}$, means that the matrix $X$ is real symmetric positive definite (resp., positive semidefinite). The symbol “*” in a matrix $A \in \mathbb{R}^{n \times n}$ stands for the transposed elements in the symmetric positions. The superscripts " $T$ " and " -1 " denote the matrix transpose and inverse, respectively. Identity matrices of appropriate dimensions will be denoted by $I$.
The shorthand $\operatorname{diag}\left\{M_{1}, M_{2}, \ldots, M_{r}\right\}$ denotes a block diagonal matrix with diagonal blocks being the matrices $M_{1}$, $M_{2}, \ldots, M_{r}$. If not explicitly stated, all matrices are assumed to have compatible dimensions for algebraic operations.

\section{Problem Formulation}

Considering the following fuzzy delta operator system with time delay, which is described by

Plant Rule i. IF $\theta_{1}\left(t_{k}\right)$ is $N_{i 1}$, and ..., and $\theta_{j}\left(t_{k}\right)$ is $N_{i j}$, and ..., and $\theta_{p}\left(t_{k}\right)$ is $N_{i p}$, THEN

$$
\begin{aligned}
& \delta x\left(t_{k}\right)=A_{i} x\left(t_{k}\right)+A_{d i} x\left(t_{k}-d_{k}\right)+B_{i} u\left(t_{k}\right)+B_{w i} w\left(t_{k}\right), \\
& z\left(t_{k}\right)=C_{i} x\left(t_{k}\right)+C_{d i} x\left(t_{k}-d_{k}\right)+D_{i} u\left(t_{k}\right)+D_{w i} w\left(t_{k}\right), \\
& x\left(t_{k}\right)=\phi\left(t_{k}\right), \quad t_{k}=-d_{M}, d_{M}+T, \ldots, 0, i=1,2, \ldots, r,
\end{aligned}
$$

where $x\left(t_{k}\right) \in \mathbb{R}^{n}$ is the state variable, $u\left(t_{k}\right) \in \mathbb{R}^{m}$ is the control input variable, $w\left(t_{k}\right) \in \mathbb{R}^{l}$ is the disturbance input variable which belongs to $L_{2}[0, \infty), z\left(t_{k}\right) \in \mathbb{R}^{p}$ is the control output, and $\phi\left(t_{k}\right)$ is a continuous vector-valued initial function. Let $t_{k}=k T$ for the convenience in analysis. $A_{i}$, $A_{d i}, B_{i}, B_{w i}, C_{i}, C_{d i}, D_{i}$, and $D_{w i}(i=1,2, \ldots, r)$ are system matrices with appropriate dimensions. The scalar $r$ is the number of IF-THEN rules. $\theta_{j}\left(t_{k}\right)$ and $N_{i j}$ are the premise variable and the fuzzy set, respectively, for $j=1,2, \ldots, p$. The parametric variable $d_{k}=n T$ which is the bounded time delay in the state and satisfies $0<d_{m} \leq d_{k} \leq$ $d_{M}$ with $d_{m}=n_{m} T$ and $d_{M}=n_{M} T\left(n_{m}\right.$ and $n_{M}$ are the known positive and finite integers, and $T$ is a sampling period). $\delta x\left(t_{k}\right)$ is the delta operator of $x\left(t_{k}\right)$, which is defined by

$$
\delta x\left(t_{k}\right)= \begin{cases}\frac{d}{d t_{k}} x\left(t_{k}\right), & T=0, \\ \frac{x\left(t_{k}+T\right)-x\left(t_{k}\right)}{T}, & T \neq 0 .\end{cases}
$$

Then the defuzzified model of system (1) is inferred as follows:

$$
\begin{array}{r}
\delta x\left(t_{k}\right)=\sum_{i=1}^{r} h_{i}\left(\theta\left(t_{k}\right)\right)\left[A_{i} x\left(t_{k}\right)+A_{d i} x\left(t_{k}-d_{k}\right)\right. \\
\left.+B_{i} u\left(t_{k}\right)+B_{w i} w\left(t_{k}\right)\right], \\
z\left(t_{k}\right)=\sum_{i=1}^{r} h_{i}\left(\theta\left(t_{k}\right)\right)\left[C_{i} x\left(t_{k}\right)+C_{d i} x\left(t_{k}-d_{k}\right)\right. \\
\left.+D_{i} u\left(t_{k}\right)+D_{w i} w\left(t_{k}\right)\right],
\end{array}
$$

where $h_{i}\left(\theta\left(t_{k}\right)\right)=\mu_{i}\left(\theta\left(t_{k}\right)\right) / \sum_{i=1}^{r} \mu_{i}\left(\theta\left(t_{k}\right)\right), \mu_{i}\left(\theta\left(t_{k}\right)\right)=$ $\prod_{j=1}^{p} N_{i j}\left(\theta_{j}\left(t_{k}\right)\right)$, and $N_{i j}\left(\theta_{j}\left(t_{k}\right)\right)$ is the degree of the membership of $\theta_{j}\left(t_{k}\right)$ in fuzzy set $N_{i j}$. It can be assumed that $\mu_{i}\left(\theta\left(t_{k}\right)\right) \geq 0($ for $i=1,2, \ldots, r)$ and $\sum_{i=1}^{r} \mu_{i}\left(\theta\left(t_{k}\right)\right) \geq 0$ (for all $t_{k}$ ). Therefore, $h_{i}\left(\theta\left(t_{k}\right)\right) \geq 0$ (for $i=1,2, \ldots, r$ ) and 
$\sum_{i=1}^{r} h_{i}\left(\theta\left(t_{k}\right)\right)=1$. Based on the parallel distributed compensation (PDC), similar to the fuzzy model, the following overall fuzzy control law can be constructed as

$$
u\left(t_{k}\right)=\sum_{s=1}^{r} h_{s}\left(\theta\left(t_{k}\right)\right) K_{s} x\left(t_{k}\right)
$$

where $K_{s}(s=1,2, \ldots, r)$ is the local control gain such that closed-loop fuzzy system (3) is asymptotically stable. Then substituting (4) into system (3), the closed-loop fuzzy system can be expressed as

$$
\begin{aligned}
\delta x\left(t_{k}\right)=\sum_{i=1}^{r} \sum_{s=1}^{r} h_{i} h_{s}[ & \left(A_{i}+B_{i} K_{s}\right) x\left(t_{k}\right) \\
& \left.+A_{d i} x\left(t_{k}-d_{k}\right)+B_{w i} w\left(t_{k}\right)\right], \\
z\left(t_{k}\right)=\sum_{i=1}^{r} \sum_{s=1}^{r} h_{i} h_{s}[ & \left(C_{i}+D_{i} K_{s}\right) x\left(t_{k}\right) \\
& \left.+C_{d i} x\left(t_{k}-d_{k}\right)+D_{w i} w\left(t_{k}\right)\right],
\end{aligned}
$$

where $\sum_{i=1}^{r} h_{i}=\sum_{i=1}^{r} h_{i}\left(\theta\left(t_{k}\right)\right)$. For the convenience, system (5) can be rewritten as

$$
\begin{aligned}
\delta x\left(t_{k}\right)= & A\left(t_{k}\right) x\left(t_{k}\right)+A_{d}\left(t_{k}\right) x\left(t_{k}-d_{k}\right) \\
& +B_{w}\left(t_{k}\right) w\left(t_{k}\right), \\
z\left(t_{k}\right)=C & \left(t_{k}\right) x\left(t_{k}\right)+C_{d}\left(t_{k}\right) x\left(t_{k}-d_{k}\right) \\
& +D_{w}\left(t_{k}\right) w\left(t_{k}\right),
\end{aligned}
$$

where

$$
\begin{aligned}
& A\left(t_{k}\right)=\sum_{i=1}^{r} \sum_{s=1}^{r} h_{i} h_{s}\left(A_{i}+B_{i} K_{s}\right), \\
& A_{d}\left(t_{k}\right)=\sum_{i=1}^{r} h_{i} A_{d i}, \quad B_{w}\left(t_{k}\right)=\sum_{i=1}^{r} h_{i} B_{w i}, \\
& C\left(t_{k}\right)=\sum_{i=1}^{r} \sum_{s=1}^{r} h_{i} h_{s}\left(C_{i}+D_{i} K_{s}\right), \\
& C_{d}\left(t_{k}\right)=\sum_{i=1}^{r} h_{i} C_{d i}, \quad D_{w}\left(t_{k}\right)=\sum_{i=1}^{r} h_{i} D_{w i} .
\end{aligned}
$$

In the next section, the following definitions and lemmas are introduced for developing the main results.

Definition 1 (see [47]). The conditions for the asymptotic stability of a delta operator system hold:

(a) $V\left(x\left(t_{k}\right)\right) \geq 0$, with equality if and only if $x\left(t_{k}\right)=0$,

(b) $\delta V\left(x\left(t_{k}\right)\right)=\left[V\left(x\left(t_{k}+T\right)\right)-V\left(x\left(t_{k}\right)\right)\right] / T<0$,

where $V\left(x\left(t_{k}\right)\right)$ is a Lyapunov function in $\delta$-domain. For Lyapunov function both in s-domain and $z$-domain, the condition (a) $V\left(x\left(t_{k}\right)\right) \geq 0$ in Definition 1 is given. On the other hand for the condition (b), when $T \rightarrow 0$, there exists

$$
\begin{aligned}
\lim _{T \rightarrow 0} \delta V\left(x\left(t_{k}\right)\right) & =\lim _{T \rightarrow 0} \frac{V\left(x\left(t_{k}+T\right)\right)-V\left(x\left(t_{k}\right)\right)}{T} \\
& =\frac{d V\left(x\left(t_{k}\right)\right)}{d t_{k}}<0,
\end{aligned}
$$

and when $T=1$, there exists

$$
\begin{aligned}
\delta V\left(x\left(t_{k}\right)\right) & =\frac{\left[V\left(x\left(t_{k}+1\right)\right)-V\left(x\left(t_{k}\right)\right)\right]}{1} \\
& =V\left(x\left(t_{k}+1\right)\right)-V\left(x\left(t_{k}\right)\right)<0 .
\end{aligned}
$$

Obviously, the Lyapunov function in $\delta$-domain can be reduced to the traditional Lyapunov function in $\mathrm{s}$-domain or $z$-domain when the sampling period $T$ tends to 0 or is 1 .

Definition 2 (see [50]). (i) System (5) is said to be passive if there exists constant $\rho$ such that

$$
2 \sum_{t_{k}=0}^{\infty} z^{T}\left(t_{k}\right) w\left(t_{k}\right) \geq \rho .
$$

(ii) System (5) is said to be strictly passive if there exist constants $\zeta>0$ and $\rho$ such that

$$
2 \sum_{t_{k}=0}^{\infty} z^{T}\left(t_{k}\right) w\left(t_{k}\right) \geq \rho+\zeta \sum_{t_{k}=0}^{\infty} w^{T}\left(t_{k}\right) w\left(t_{k}\right) .
$$

(iii) System (5) is said to be output strictly passive if there exist constants $\varepsilon>0$ and $\rho$ such that

$$
2 \sum_{t_{k}=0}^{\infty} z^{T}\left(t_{k}\right) w\left(t_{k}\right) \geq \rho+\varepsilon \sum_{t_{k}=0}^{\infty} z^{T}\left(t_{k}\right) z\left(t_{k}\right) .
$$

(iv) System (5) is said to be very strictly passive if there exist constants $\varepsilon>0, \zeta>0$, and $\rho$ such that

$$
\begin{aligned}
2 \sum_{t_{k}=0}^{\infty} z^{T}\left(t_{k}\right) w\left(t_{k}\right) \geq & \rho \varepsilon \sum_{t_{k}=0}^{\infty} z^{T}\left(t_{k}\right) z\left(t_{k}\right) \\
& +\zeta \sum_{t_{k}=0}^{\infty} w^{T}\left(t_{k}\right) w\left(t_{k}\right) .
\end{aligned}
$$

Lemma 3 (see [47]). For any of the time functions $x\left(t_{k}\right)$ and $y\left(t_{k}\right)$,

$$
\begin{aligned}
\delta\left(x\left(t_{k}\right) y\left(t_{k}\right)\right)= & \delta\left(x\left(t_{k}\right)\right) y\left(t_{k}\right)+x\left(t_{k}\right) \delta\left(y\left(t_{k}\right)\right) \\
& +T \delta\left(x\left(t_{k}\right)\right) \delta\left(y\left(t_{k}\right)\right),
\end{aligned}
$$

where $T$ is a sampling period.

Lemma 4 (see [51]). For any of the two positive integers $r$ and $r_{0}$, satisfying $1 \leq r_{0} \leq r$ holds:

$$
\left[\sum_{i=r_{0}}^{r} x(i)\right]^{T} M\left[\sum_{i=r_{0}}^{r} x(i)\right] \leq\left(r-r_{0}+1\right) \sum_{i=r_{0}}^{r} x^{T}(i) M x(i),
$$

where $M$ is a constant positive semidefinite symmetric matrix. 
Lemma 5 (see [52]). For the given constant matrices $G, H$ and a symmetric constant matrix $\chi$ of appropriate dimensions, the following inequality holds:

$$
\chi+G F\left(t_{k}\right) H+H^{T} F^{T}\left(t_{k}\right) G^{T} \leq 0
$$

where $F\left(t_{k}\right)$ satisfies $F^{T}\left(t_{k}\right) F\left(t_{k}\right) \leq I$, if and only if for $\xi>0$ the following inequality holds:

$$
\chi+\xi^{-1} H^{T} H+\xi G G^{T} \leq 0 .
$$

Lemma 6. If there exist scalars $\varepsilon>0, \zeta>0$, and a differential function $V\left(x\left(t_{k}\right)\right) \geq 0$ such that

$$
\begin{gathered}
\delta V\left(x\left(t_{k}\right)\right)+\varepsilon z^{T}\left(t_{k}\right) z\left(t_{k}\right)+\zeta w^{T}\left(t_{k}\right) w\left(t_{k}\right) \\
-2 z^{T}\left(t_{k}\right) w\left(t_{k}\right) \leq 0,
\end{gathered}
$$

then system (5) is very strictly passive.

Proof. It follows from (18) that

$$
\begin{aligned}
2 \sum_{t_{k}=0}^{\infty} z^{T}\left(t_{k}\right) w\left(t_{k}\right) \geq & -V(x(0))+\varepsilon \sum_{t_{k}=0}^{\infty} z^{T}\left(t_{k}\right) z\left(t_{k}\right) \\
& +\zeta \sum_{t_{k}=0}^{\infty} w^{T}\left(t_{k}\right) w\left(t_{k}\right) \\
= & \rho+\varepsilon \sum_{t_{k}=0}^{\infty} z^{T}\left(t_{k}\right) z\left(t_{k}\right) \\
& +\zeta \sum_{t_{k}=0}^{\infty} w^{T}\left(t_{k}\right) w\left(t_{k}\right)
\end{aligned}
$$

where $\rho=-V(x(0))$. Then, it can be seen that (13) is equivalent to (18) for very strict passive definition.

Remark 7. Based on Definition 2, the main objective of this paper is just to prove that T-S fuzzy system (5) is very strictly passive via delta operator approach, which can also satisfy other three indexs. The very strictly passive control for system (5) is shown in the next section.

\section{Main Results}

This section focuses on designing a sufficient condition for the solvability of the proposed passive control problem and a developed LMI approach for designing the passive controller for fuzzy system (5). Firstly, the passivity analysis criterion is derived for the system (5) in the following theorem.

Theorem 8. Considering fuzzy delta operator system (5), for a given sampling period $T>0$, constants $d_{m}, d_{M}\left(0<d_{m} \leq d_{M}\right)$, and matrix $K_{s}(s=1,2, \ldots, r)$, system (5) is very strictly passive if there exist scalars $\varepsilon>0, \zeta>0$ and symmetric matrices $P>0, R_{1}>0, R_{2}>0, Q_{\kappa}>0, S_{1 \kappa}>0$, and $S_{2 \kappa}>0$ $(\kappa=1,2, \ldots, r)$ with appropriate dimensions, such that the following LMIs hold for $\kappa, \lambda, \mu, \nu, i, s=1,2, \ldots, r$ :

$$
\begin{gathered}
\Theta_{\kappa \lambda \mu v i i}<0, \\
\Theta_{\kappa \lambda \mu \nu i s}+\Theta_{\kappa \lambda \mu \nu s i}<0, \quad s \neq i,
\end{gathered}
$$

where

$$
\begin{aligned}
& \Theta_{\kappa \lambda \mu \nu i s}=\left[\begin{array}{cc}
\Omega_{1 \kappa \lambda \mu \nu i s} & \Omega_{2 i s}^{T} \\
* & -\varepsilon I
\end{array}\right], \quad \Omega_{2 i s}=\left[\begin{array}{llllll}
0 & C_{i}+D_{i} K_{s} & C_{d i} & 0 & 0 & D_{w i}
\end{array}\right], \\
& \Omega_{1 \kappa \lambda \mu v i s}=\left[\begin{array}{cccccc}
\Xi_{1} & P\left(A_{i}+B_{i} K_{s}\right) & P A_{d i} & 0 & 0 & P B_{w i} \\
* & \Xi_{2 \kappa i s} & P A_{d i} & \frac{1}{d_{m}} R_{1} & \frac{1}{d_{M}} R_{2} & \Xi_{3 i s} \\
* & * & -Q_{\lambda} & 0 & 0 & -C_{d i} \\
* & * & * & -S_{1 \mu}-\frac{1}{d_{m}} R_{1} & 0 & 0 \\
* & * & * & * & -S_{2 v}-\frac{1}{d_{M}} R_{2} & 0 \\
* & * & * & * & * & \zeta I-D_{w i}-D_{w i}^{T}
\end{array}\right], \\
& \Xi_{1}=(T-2) P+d_{m} R_{1}+d_{M} R_{2}, \quad \Xi_{3 i s}=P B_{w i}-\left(C_{i}+D_{i} K_{s}\right)^{T}, \\
& \Xi_{2 \kappa i s}=P\left(A_{i}+B_{i} K_{s}\right)+\left(A_{i}+B_{i} K_{s}\right)^{T} P+\left(d_{M}-d_{m}+T+1\right) Q_{\kappa}+S_{1 \kappa}+S_{2 \kappa}-\frac{1}{d_{m}} R_{1}-\frac{1}{d_{M}} R_{2} .
\end{aligned}
$$


Proof. Firstly, in order to simplify the calculation, relevant fuzzy weighing matrices which directly include the membership functions are defined as follows:

$$
\begin{aligned}
& \breve{Q}\left(t_{k}\right)=\sum_{\kappa=1}^{r} h_{\kappa}\left(\theta\left(t_{k}\right)\right) Q_{\kappa}, \quad \breve{S}_{1}\left(t_{k}\right)=\sum_{\kappa=1}^{r} h_{\kappa}\left(\theta\left(t_{k}\right)\right) S_{1 \kappa}, \\
& \breve{S}_{2}\left(t_{k}\right)=\sum_{\kappa=1}^{r} h_{\kappa}\left(\theta\left(t_{k}\right)\right) S_{2 \kappa} .
\end{aligned}
$$

Choose a LKF $V\left(x\left(t_{k}\right)\right)$ for system (5) as follows:

$$
\begin{aligned}
V\left(x\left(t_{k}\right)\right)= & V_{1}\left(x\left(t_{k}\right)\right)+V_{2}\left(x\left(t_{k}\right)\right)+V_{3}\left(x\left(t_{k}\right)\right) \\
& +V_{4}\left(x\left(t_{k}\right)\right)+V_{5}\left(x\left(t_{k}\right)\right),
\end{aligned}
$$

where

$$
\begin{aligned}
V_{1}\left(x\left(t_{k}\right)\right)= & x^{T}\left(t_{k}\right) P x\left(t_{k}\right), \\
V_{2}\left(x\left(t_{k}\right)\right)= & T \sum_{i=1}^{n} x^{T}\left(t_{k}-i T\right) \breve{Q}\left(t_{k}-i T\right) x\left(t_{k}-i T\right), \\
V_{3}\left(x\left(t_{k}\right)\right)= & T \sum_{i=1}^{n_{m}} x^{T}\left(t_{k}-i T\right) \breve{S}_{1}\left(t_{k}-i T\right) x\left(t_{k}-i T\right) \\
& +T \sum_{i=1}^{n_{M}} x^{T}\left(t_{k}-i T\right) \breve{S}_{2}\left(t_{k}-i T\right) x\left(t_{k}-i T\right), \\
V_{4}\left(x\left(t_{k}\right)\right)= & T^{2} \sum_{i=n_{m}}^{n_{M}} \sum_{s=1}^{i} x^{T}\left(t_{k}-s T\right) \breve{Q}\left(t_{k}-s T\right) x\left(t_{k}-s T\right), \\
V_{5}\left(x\left(t_{k}\right)\right)= & \sum_{i=1}^{n_{m}} \sum_{s=1}^{i} e^{T}\left(t_{k}-s T\right) R_{1} e\left(t_{k}-s T\right) \\
& +\sum_{i=1}^{n_{M}} \sum_{s=1}^{i} e^{T}\left(t_{k}-s T\right) R_{2} e\left(t_{k}-s T\right),
\end{aligned}
$$

and symmetric matrices $P>0, R_{1}>0, R_{2}>0, Q_{\kappa}>0, S_{1 \kappa}>$ $0, S_{2 \kappa}>0($ for $\kappa=1,2, \ldots, r)$, and $e(s)=x(s)-x(s+T)$ from which it can be concluded that there exist $e\left(t_{k}-i T\right)=$ $x\left(t_{k}-i T\right)-x\left(t_{k}-(i-1) T\right)$ and $\delta x\left(t_{k}\right)=-e\left(t_{k}\right) / T$.

Applying Lemma 3 to $V_{1}\left(x\left(t_{k}\right)\right)$ and along the trajectory of the system (5), it can be obtained that

$$
\begin{aligned}
\delta V_{1}\left(x\left(t_{k}\right)\right)= & \delta^{T}\left(x\left(t_{k}\right)\right) P x\left(t_{k}\right)+x^{T}\left(t_{k}\right) P \delta\left(x\left(t_{k}\right)\right) \\
& +T \delta^{T}\left(x\left(t_{k}\right)\right) P \delta\left(x\left(t_{k}\right)\right) \\
= & x^{T}\left(t_{k}\right)\left[P A\left(t_{k}\right)+A^{T}\left(t_{k}\right) P\right] x\left(t_{k}\right) \\
& +2 x^{T}\left(t_{k}\right) P A_{d}\left(t_{k}\right) x\left(t_{k}-d_{k}\right)
\end{aligned}
$$

$$
\begin{aligned}
& +2 x^{T}\left(t_{k}\right) P B_{w}\left(t_{k}\right) w\left(t_{k}\right) \\
& +T \delta^{T}\left(x\left(t_{k}\right)\right) P \delta\left(x\left(t_{k}\right)\right) .
\end{aligned}
$$

Similarly, applying the delta operator to $V_{2}\left(x\left(t_{k}\right)\right)$, $V_{3}\left(x\left(t_{k}\right)\right)$, and $V_{4}\left(x\left(t_{k}\right)\right)$, it can be obtained as follows:

$$
\begin{aligned}
& \delta V_{2}\left(x\left(t_{k}\right)\right)=\frac{1}{T}\left[T \sum_{i=1}^{n} x^{T}\left(t_{k}-i T+T\right) \breve{Q}\left(t_{k}-i T+T\right)\right. \\
& \times x\left(t_{k}-i T+T\right) \\
& \left.-T \sum_{i=1}^{n} x^{T}\left(t_{k}-i T\right) \breve{Q}\left(t_{k}-i T\right) x\left(t_{k}-i T\right)\right] \\
& \leq x^{T}\left(t_{k}\right) \breve{Q}\left(t_{k}\right) x\left(t_{k}\right) \\
& -x^{T}\left(t_{k}-d_{k}\right) \breve{Q}\left(t_{k}-d_{k}\right) x\left(t_{k}-d_{k}\right) \\
& +T \sum_{i=n_{m}}^{n_{M}} x^{T}\left(t_{k}-i T\right) \breve{Q}\left(t_{k}-i T\right) x\left(t_{k}-i T\right), \\
& \delta V_{3}\left(x\left(t_{k}\right)\right)=x^{T}\left(t_{k}\right)\left(\breve{S}_{1}\left(t_{k}\right)+\breve{S}_{2}\left(t_{k}\right)\right) x\left(t_{k}\right) \\
& -x^{T}\left(t_{k}-d_{m}\right) \breve{S}_{1}\left(t_{k}-d_{m}\right) x\left(t_{k}-d_{m}\right) \\
& -x^{T}\left(t_{k}-d_{M}\right) S_{2}\left(t_{k}-d_{M}\right) x\left(t_{k}-d_{M}\right), \\
& \delta V_{4}\left(x\left(t_{k}\right)\right)=T\left[\sum _ { s = 1 } ^ { i } \left(x^{T}\left(t_{k}-s T+T\right) \breve{Q}\left(t_{k}-s T+T\right)\right.\right. \\
& \left.\times x\left(t_{k}-s T+T\right)\right) \\
& -\sum_{s=1}^{i}\left(x^{T}\left(t_{k}-s T\right) \breve{Q}\left(t_{k}-s T\right)\right. \\
& \left.\left.\times x\left(t_{k}-s T\right)\right)\right] \\
& =\left(d_{M}-d_{m}+T\right) x^{T}\left(t_{k}\right) \breve{Q}\left(t_{k}\right) x\left(t_{k}\right) \\
& -T \sum_{i=n_{m}}^{n_{M}} x^{T}\left(t_{k}-i T\right) \breve{Q}\left(t_{k}-i T\right) x\left(t_{k}-i T\right) \text {. }
\end{aligned}
$$

Applying Lemma 4 to $V_{5}\left(x\left(t_{k}\right)\right)$ and along the trajectory of system (5), it can be obtained that

$$
\begin{aligned}
& \delta V_{5}\left(x\left(t_{k}\right)\right)=\frac{1}{T}\left[\sum_{i=1}^{n_{m}} \sum_{s=1}^{i} e^{T}\left(t_{k}-s T+T\right) R_{1} e\left(t_{k}-s T+T\right)\right. \\
&-\sum_{i=1}^{n_{m}} \sum_{s=1}^{i} e^{T}\left(t_{k}-s T\right) R_{1} e\left(t_{k}-s T\right) \\
&+\sum_{i=1}^{n_{M}} \sum_{s=1}^{i} e^{T}\left(t_{k}-s T+T\right)
\end{aligned}
$$




$$
\begin{aligned}
& \times R_{2} e\left(t_{k}-s T+T\right) \\
& \left.-\sum_{i=1}^{n_{M}} \sum_{s=1}^{i} e^{T}\left(t_{k}-s T\right) R_{2} e\left(t_{k}-s T\right)\right] \\
& =\frac{1}{T}\left[\sum_{i=1}^{n_{m}} e^{T}\left(t_{k}\right) R_{1} e\left(t_{k}\right)\right. \\
& -\sum_{i=1}^{n_{m}} e^{T}\left(t_{k}-i T\right) R_{1} e\left(t_{k}-i T\right) \\
& +\sum_{i=1}^{n_{M}} e^{T}\left(t_{k}\right) R_{2} e\left(t_{k}\right) \\
& \left.-\sum_{i=1}^{n_{M}} e^{T}\left(t_{k}-i T\right) R_{2} e\left(t_{k}-i T\right)\right] \\
& \leq e^{T}\left(t_{k}\right)\left(\frac{n_{m}}{T} R_{1}+\frac{n_{M}}{T} R_{2}\right) e\left(t_{k}\right) \\
& -\frac{1}{n_{m} T}\left[\sum_{i=1}^{n_{m}} e\left(t_{k}-i T\right)\right]^{T} R_{1} \\
& \times\left[\sum_{i=1}^{n_{m}} e\left(t_{k}-i T\right)\right]-\frac{1}{n_{M} T}\left[\sum_{i=1}^{n_{M}} e\left(t_{k}-i T\right)\right]^{T} \\
& \times R_{2}\left[\sum_{i=1}^{n_{M}} e\left(t_{k}-i T\right)\right] \\
& =\delta^{T}\left(x\left(t_{k}\right)\right)\left(d_{m} R_{1}+d_{M} R_{2}\right) \delta\left(x\left(t_{k}\right)\right) \\
& -\frac{1}{d_{m}}\left[x\left(t_{k}\right)-x\left(t_{k}-d_{m}\right)\right]^{T} \\
& \times R_{1}\left[x\left(t_{k}\right)-x\left(t_{k}-d_{m}\right)\right] \\
& -\frac{1}{d_{M}}\left[x\left(t_{k}\right)-x\left(t_{k}-d_{M}\right)\right]^{T} \\
& \times R_{2}\left[x\left(t_{k}\right)-x\left(t_{k}-d_{M}\right)\right] .
\end{aligned}
$$

For the real matrix $P>0$, the following equation is tenable:

$$
\begin{aligned}
0= & -2 \delta^{T}\left(x\left(t_{k}\right)\right) P\left[\delta\left(x\left(t_{k}\right)\right)-A\left(t_{k}\right) x\left(t_{k}\right)\right. \\
& \left.-A_{d}\left(t_{k}\right) x\left(t_{k}-d_{k}\right)-B_{w}\left(t_{k}\right) w\left(t_{k}\right)\right] \\
= & -2 \delta^{T}\left(x\left(t_{k}\right)\right) P \delta\left(x\left(t_{k}\right)\right)+2 \delta^{T}\left(x\left(t_{k}\right)\right) P A\left(t_{k}\right) x\left(t_{k}\right) \\
& +2 \delta^{T}\left(x\left(t_{k}\right)\right) P A_{d}\left(t_{k}\right) x\left(t_{k}-d_{k}\right) \\
& +2 \delta^{T}\left(x\left(t_{k}\right)\right) P B_{w}\left(t_{k}\right) w\left(t_{k}\right) .
\end{aligned}
$$

Generally, considering the passive performance index in Definition 2, which is described as the passivity analysis, performance of system (5) can be established as follows:

$$
\begin{aligned}
J= & \varepsilon^{-1} z^{T}\left(t_{k}\right) z\left(t_{k}\right)+\zeta w^{T}\left(t_{k}\right) w\left(t_{k}\right) \\
& -2 z^{T}\left(t_{k}\right) w\left(t_{k}\right)+\delta V\left(t_{k}\right) .
\end{aligned}
$$

By adding (28) into $\delta V\left(t_{k}\right)$ and applying (7), it can be found that

$$
\begin{aligned}
J= & \varepsilon^{-1} z^{T}\left(t_{k}\right) z\left(t_{k}\right)+\zeta w^{T}\left(t_{k}\right) w\left(t_{k}\right)-2 z^{T}\left(t_{k}\right) w\left(t_{k}\right) \\
& +\delta V\left(t_{k}\right) \\
\leq & \eta^{T}\left(t_{k}\right) \sum_{\kappa=1}^{r} \sum_{\lambda=1}^{r} \sum_{\mu=1}^{r} \sum^{r} \sum^{r} \sum_{i=1}^{r} \sum_{s=1}^{r} h_{\kappa} h_{\lambda} h_{\mu} h_{v} h_{i} h_{s} \eta^{T}\left(t_{k}\right) \\
& \times\left[\Omega_{1 \kappa \lambda \mu v i s}+\varepsilon^{-1} \Omega_{2 i s}^{T} \Omega_{2 i s}\right] \eta\left(t_{k}\right),
\end{aligned}
$$

where $h_{\kappa}=h_{\kappa}\left(\theta\left(t_{k}\right)\right), h_{\lambda}=h_{\lambda}\left(\theta\left(t_{k}-d_{k}\right)\right), h_{\mu}=h_{\mu}\left(\theta\left(t_{k}-\right.\right.$ $\left.\left.d_{m}\right)\right), h_{v}=h_{v}\left(\theta\left(t_{k}-d_{M}\right)\right), h_{i}=h_{i}\left(\theta\left(t_{k}\right)\right), h_{s}=h_{s}\left(\theta\left(t_{k}\right)\right)$, and

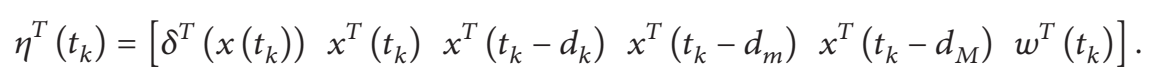

On the other hand, it can be seen from Theorem 8 that

$$
\begin{aligned}
\sum_{\kappa=1}^{r} \sum_{\lambda=1}^{r} \sum_{\mu=1}^{r} \sum_{\nu=1}^{r} \sum_{i=1}^{r} \sum_{s=1}^{r} h_{\kappa} h_{\lambda} h_{\mu} h_{\nu} h_{i} h_{s} \Theta_{\kappa \lambda \mu v i s} \\
=\sum_{\kappa=1}^{r} \sum_{\lambda=1}^{r} \sum_{\mu=1}^{r} \sum_{v=1}^{r} h_{\kappa} h_{\lambda} h_{\mu} h_{\nu} \\
\times\left[\sum_{i=1}^{r} h_{i}^{2} \Theta_{\kappa \lambda \mu \nu i i}\right.
\end{aligned}
$$

$$
\left.+\sum_{i=1}^{r-1} \sum_{s=i+1}^{r} h_{i} h_{s}\left(\Theta_{\kappa \lambda \mu v i s}+\Theta_{\kappa \lambda \mu \nu s i}\right)\right]<0
$$

Applying Schur complement, it can be obtained that

$$
\begin{gathered}
J=\varepsilon^{-1} z^{T}\left(t_{k}\right) z\left(t_{k}\right)+\zeta w^{T}\left(t_{k}\right) w\left(t_{k}\right) \\
-2 z^{T}\left(t_{k}\right) w\left(t_{k}\right)+\delta V\left(t_{k}\right)<0 .
\end{gathered}
$$


It can be seen that the LMIs conditions in Theorem 8 satisfy the very strictly passive performance index for the fuzzy delta operator system (5). The proof is completed.

Based on the conditions in Theorem 8 , the state-feedback control gain matrices $K_{s}(s=1,2, \ldots, r)$ will be designed in the following theorem.

Theorem 9. Considering fuzzy delta operator system (5), for a given sampling period $T>0$ and constants $d_{m}, d_{M}(0<$ $d_{m} \leq d_{M}$ ), system (5) is very strictly passive if there exist scalars $\varepsilon>0, \zeta>0$ and symmetric matrices $\bar{P}>0, \bar{R}_{1}>0$, $\bar{R}_{2}>0, \bar{Q}_{\kappa}>0, \bar{S}_{1 \kappa}>0$, and $\bar{S}_{2 \kappa}>0(\kappa=1,2, \ldots, r)$ with appropriate dimensions, such that the following LMIs hold for $\kappa, \lambda, \mu, \nu, i, s=1,2, \ldots, r$ :

$$
\begin{gathered}
\bar{\Theta}_{\kappa \lambda \mu v i i}<0, \\
\bar{\Theta}_{\kappa \lambda \mu v i s}+\bar{\Theta}_{\kappa \lambda \mu \nu s i}<0, \quad s \neq i,
\end{gathered}
$$

where

$$
\begin{aligned}
& \bar{\Theta}_{\kappa \lambda \mu v i s}=\left[\begin{array}{cc}
\bar{\Omega}_{1 \kappa \lambda \mu v i s} & \bar{\Omega}_{2 i s}^{T} \\
* & -\varepsilon I
\end{array}\right], \quad \bar{\Omega}_{2 \lambda \mu v i s}=\left[\begin{array}{llllll}
0 & \bar{C}_{i} \bar{P}+D_{i} \bar{K}_{s} & C_{d i} \bar{P} & 0 & 0 & D_{w i}
\end{array}\right], \\
& \bar{\Omega}_{1 \kappa \lambda \mu v i s}=\left[\begin{array}{cccccc}
\bar{\Xi}_{1} & A_{i} \bar{P}+B_{i} \bar{K}_{s} & A_{d i} \bar{P} & 0 & 0 & B_{w i} \\
* & \bar{\Xi}_{2 \kappa i s} & A_{d i} \bar{P} & \frac{1}{d_{m}} \bar{R}_{1} & \frac{1}{d_{M}} \bar{R}_{2} & \bar{\Xi}_{3 i s} \\
* & * & -\bar{Q}_{\lambda} & 0 & 0 & -\bar{P} C_{d i}^{T} \\
* & * & * & -\bar{S}_{1 \mu}-\frac{1}{d_{m}} \bar{R}_{1} & 0 & 0 \\
* & * & * & * & -\bar{S}_{2 v}-\frac{1}{d_{M}} \bar{R}_{2} & 0 \\
* & * & * & * & * & \zeta I-D_{w i}-D_{w i}^{T}
\end{array}\right], \\
& \bar{\Xi}_{1}=(T-2) \bar{P}+d_{m} \bar{R}_{1}+d_{M} \bar{R}_{2}, \quad \bar{\Xi}_{3 i s}=B_{w i}-\bar{P} C_{i}^{T}-\bar{K}_{s}^{T} D_{i}^{T}, \\
& \bar{\Xi}_{2 \kappa i s}=A_{i} \bar{P}+\bar{P} A_{i}^{T}+B_{i} \bar{K}_{s}+\bar{K}_{s}^{T} B_{i}^{T}+\left(d_{M}-d_{m}+T+1\right) \bar{Q}_{\kappa}+\bar{S}_{1 \kappa}+\bar{S}_{2 \kappa}-\frac{1}{d_{m}} \bar{R}_{1}-\frac{1}{d_{M}} \bar{R}_{2} .
\end{aligned}
$$

The fuzzy sate-feedback controller can be obtained:

$$
u\left(t_{k}\right)=\bar{K}_{s} \bar{P}^{-1} x\left(t_{k}\right) .
$$

Proof. Premultiplying and postmultiplying (34) by diagonal matrix

$$
\operatorname{diag}\left\{\bar{P}^{-1}, \bar{P}^{-1}, \bar{P}^{-1}, \bar{P}^{-1}, \bar{P}^{-1}, I, I\right\}
$$

and letting

$$
\begin{gathered}
P=\bar{P}^{-1}, \quad K_{s}=\bar{K}_{s} \bar{P}^{-1}, \quad R_{1}=\bar{P}^{-1} \bar{R}_{1} \bar{P}^{-1}, \\
R_{2}=\bar{P}^{-1} \bar{R}_{2} \bar{P}^{-1}, \\
Q_{i}=\bar{P}^{-1} \bar{Q}_{i} \bar{P}^{-1}, \quad S_{1 i}=\bar{P}^{-1} \bar{S}_{1 i} \bar{P}^{-1} \\
S_{2 i}=\bar{P}^{-1} \bar{S}_{2 i} \bar{P}^{-1}
\end{gathered}
$$

for $i=1,2, \ldots, r$, it can be found that the conditions (20) hold, which means that system (5) is very strictly passive. The proof is completed.
Remark 10. In this paper, the modeling uncertainties are not taken into account in system (1). It should be pointed out that the robust passive controller design condition can be presented for uncertain discrete-time T-S fuzzy systems with time delay and disturbance input via delta operator approach.

In order to present the stability and stabilization condition for discrete-time T-S fuzzy systems with time delay via delta operator approach, it is assumed that the disturbance input $w\left(t_{k}\right)=0$ in fuzzy delta operator system (3), which can be described as

$$
\begin{aligned}
& \delta x\left(t_{k}\right)=\sum_{i=1}^{r} h_{i}\left(\theta\left(t_{k}\right)\right) \\
& \times\left[A_{i} x\left(t_{k}\right)+A_{d i} x\left(t_{k}-d_{k}\right)+B_{i} u\left(t_{k}\right)\right], \\
& z\left(t_{k}\right)=\sum_{i=1}^{r} h_{i}\left(\theta\left(t_{k}\right)\right) \\
& \times\left[C_{i} x\left(t_{k}\right)+C_{d i} x\left(t_{k}-d_{k}\right)+D_{i} u\left(t_{k}\right)\right] .
\end{aligned}
$$


Similar to the proof of Theorems 8 and 9, the following two corollaries can be obtained. with appropriate dimensions, such that the following LMIs hold for $\kappa, \lambda, \mu, \nu, i=1,2, \ldots, r$ :

Corollary 11. For a given sampling period $T>0$ and constants $d_{m}$ and $d_{M}\left(0<d_{m} \leq d_{M}\right)$, system (39) $\left(u\left(t_{k}\right)=0\right)$ is asymptotically stable if there exist symmetric matrices $P>0$, $R_{1}>0, R_{2}>0, Q_{\kappa}>0, S_{1 \kappa}>0$, and $S_{2 \kappa}>0(\kappa=1,2, \ldots, r)$

$$
\breve{\Theta}_{\kappa \lambda \mu \nu i}<0 \text {, }
$$

where

$$
\begin{gathered}
\breve{\Theta}_{\kappa \lambda \mu v i}=\left[\begin{array}{ccccc}
\Xi_{1} & P A_{i} & P A_{d i} & 0 & 0 \\
* & \breve{\Xi}_{2 \kappa i} & P A_{d i} & \frac{1}{d_{m}} R_{1} & \frac{1}{d_{M}} R_{2} \\
* & * & -Q_{\lambda} & 0 & 0 \\
* & * & * & -S_{1 \mu}-\frac{1}{d_{m}} R_{1} & 0 \\
* & * & * & * & -S_{2 v}-\frac{1}{d_{M}} R_{2}
\end{array}\right], \\
\breve{\Xi}_{2 \kappa i}=P A_{i}+A_{i}^{T} P+\left(d_{M}-d_{m}+T+1\right) Q_{\kappa}+S_{1 \kappa}+S_{2 \kappa}-\frac{1}{d_{m}} R_{1}-\frac{1}{d_{M}} R_{2} .
\end{gathered}
$$

Corollary 12. System (39) with the controller in (4) is asymptotically stable for a given sampling period $T>0$ and constants $d_{m}$ and $d_{M}\left(0<d_{m} \leq d_{M}\right)$, if there exist symmetric matrices $\bar{P}>0, \bar{R}_{1}>0, \bar{R}_{2}>0, \bar{Q}_{\kappa}>0, \bar{S}_{1 \kappa}>0$, and $\bar{S}_{2 \kappa}>0$ $(\kappa=1,2, \ldots, r)$ with appropriate dimensions, such that the following LMIs hold for $\kappa, \lambda, \mu, \nu, i, s=1,2, \ldots, r$ :

$$
\begin{gathered}
\widetilde{\Theta}_{\kappa \lambda \mu \nu i i}<0, \\
\widetilde{\Theta}_{\kappa \lambda \mu \nu i s}+\widetilde{\Theta}_{\kappa \lambda \mu \nu s i}<0, \quad s \neq i,
\end{gathered}
$$

where

$$
\begin{gathered}
\widetilde{\Theta}_{\kappa \lambda \mu \nu i s}=\left[\begin{array}{ccccc}
\bar{\Xi}_{1} & A_{i} \bar{P}+B_{i} \bar{K}_{s} & A_{d i} \bar{P} & 0 & 0 \\
* & \bar{\Xi}_{2 \kappa i s} & A_{d i} \bar{P} & \frac{1}{d_{m}} \bar{R}_{1} & \frac{1}{d_{M}} \bar{R}_{2} \\
* & * & -\bar{Q}_{\lambda} & 0 & 0 \\
* & * & * & -\bar{S}_{1 \mu}-\frac{1}{d_{m}} \bar{R}_{1} & 0 \\
* & * & * & * & -\bar{S}_{2 v}-\frac{1}{d_{M}} \bar{R}_{2}
\end{array}\right], \\
\bar{\Xi}_{2 \kappa i s}=A_{i} \bar{P}+\bar{P} A_{i}^{T}+B_{i} \bar{K}_{s}+\bar{K}_{s}^{T} B_{i}^{T}+\left(d_{M}-d_{m}+T+1\right) \bar{Q}_{\kappa}+\bar{S}_{1 \kappa}+\bar{S}_{2 \kappa}-\frac{1}{d_{m}} \bar{R}_{1}-\frac{1}{d_{M}} \bar{R}_{2} .
\end{gathered}
$$

Then, the controller (4) can be designed as

$$
u\left(t_{k}\right)=\bar{K}_{s} \bar{P}^{-1} x\left(t_{k}\right) .
$$

\section{Numerical Example}

In this section, one example is presented to demonstrate the effectiveness of the proposed method.
Example 1. Consider the following fuzzy system with timevarying delay and input disturbance:

Plant Rule 1. IF $\theta\left(t_{k}\right)$ is 0 , THEN

$$
\begin{aligned}
\delta x\left(t_{k}\right)= & A_{1} x\left(t_{k}\right)+A_{d 1} x\left(t_{k}-d_{k}\right)+B_{1} u\left(t_{k}\right) \\
& +B_{w 1} w\left(t_{k}\right),
\end{aligned}
$$


TABLE 1: The feasibility of the system with $T=0.001$.

\begin{tabular}{llllll}
\hline & $d_{m}$ & $d_{M}$ & $K_{1}$ & $K_{2}$ & \\
\hline$T=0.001$ & 0.01 & 7.44 & {$\left[\begin{array}{lll}-0.2229 & -13.6801\end{array}\right]$} & {$[-1.7743$} & $-4.5044]$ \\
\hline
\end{tabular}

$$
\begin{gathered}
z\left(t_{k}\right)=C_{1} x\left(t_{k}\right)+C_{d 1} x\left(t_{k}-d_{k}\right)+D_{1} u\left(t_{k}\right) \\
+D_{w 1} w\left(t_{k}\right) .
\end{gathered}
$$

Plant Rule 2. IF $\theta\left(t_{k}\right)$ is $\pm(\pi / 2)\left(\left|\theta\left(t_{k}\right)\right|<\pi / 2\right)$, THEN

$$
\begin{aligned}
& \delta x\left(t_{k}\right)= A_{2} x\left(t_{k}\right)+A_{d 2} x\left(t_{k}-d_{k}\right)+B_{2} u\left(t_{k}\right) \\
&+B_{w 2} w\left(t_{k}\right), \\
& z\left(t_{k}\right)=C_{2} x\left(t_{k}\right)+C_{d 2} x\left(t_{k}-d_{k}\right)+D_{2} u\left(t_{k}\right) \\
&+D_{w 2} w\left(t_{k}\right) .
\end{aligned}
$$

The system matrix parameters in (45) and (46) are given as follows:

$$
\begin{aligned}
& A_{1}=\left[\begin{array}{cc}
-2.000 & -0.060 \\
0.070 & 0.100
\end{array}\right], \quad A_{2}=\left[\begin{array}{cc}
-1.800 & 0.080 \\
-0.050 & 0.100
\end{array}\right], \\
& B_{1}=\left[\begin{array}{l}
0.080 \\
0.050
\end{array}\right], \quad B_{2}=\left[\begin{array}{l}
0.080 \\
0.090
\end{array}\right], \\
& A_{d 1}=\left[\begin{array}{cc}
-0.020 & -0.010 \\
0.010 & 0.020
\end{array}\right], \quad A_{d 2}=\left[\begin{array}{ll}
-1.800 & 0.080 \\
-0.050 & 0.100
\end{array}\right], \\
& B_{w 1}=\left[\begin{array}{ll}
0.010 & -0.100
\end{array}\right]^{T}, \\
& B_{w 2}=\left[\begin{array}{ll}
-0.010 & -0.100
\end{array}\right]^{T}, \quad C_{1}=\left[\begin{array}{ll}
-0.010 & 0.010
\end{array}\right], \\
& C_{2}=\left[\begin{array}{ll}
0.120 & -0.100
\end{array}\right], \quad D_{1}=0.010, \\
& C_{d 1}=\left[\begin{array}{ll}
0.080 & 0.010
\end{array}\right], \quad C_{d 2}=\left[\begin{array}{ll}
-0.010 & -0.050
\end{array}\right], \\
& D_{2}=0.020, \quad D_{w 1}=0.010, \quad D_{w 2}=0.020 .
\end{aligned}
$$

Two membership functions are chosen for Plant Rules 1 and 2 as follows:

$$
\begin{gathered}
h_{1}\left(\theta\left(t_{k}\right)\right)= \begin{cases}1-\frac{2}{\pi} \theta\left(t_{k}\right), & 0 \leq \theta\left(t_{k}\right) \leq \frac{\pi}{2}, \\
1+\frac{2}{\pi} \theta\left(t_{k}\right), & -\frac{\pi}{2} \leq \theta\left(t_{k}\right)<0,\end{cases} \\
h_{2}\left(\theta\left(t_{k}\right)\right)=1-h_{1}\left(\theta\left(t_{k}\right)\right) .
\end{gathered}
$$

And Figure 1 shows the membership functions.

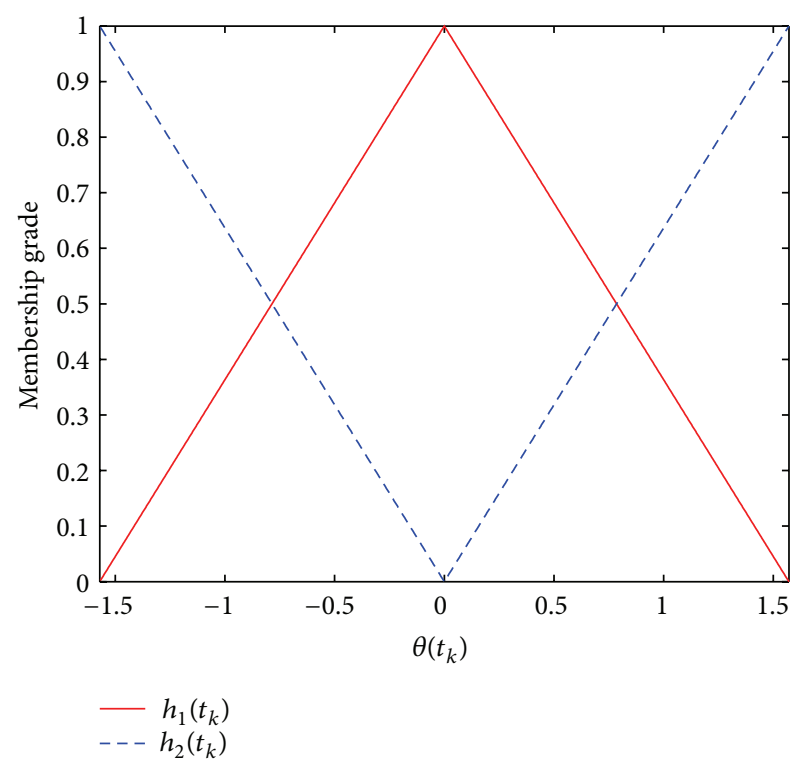

Figure 1: Membership functions of two rules.

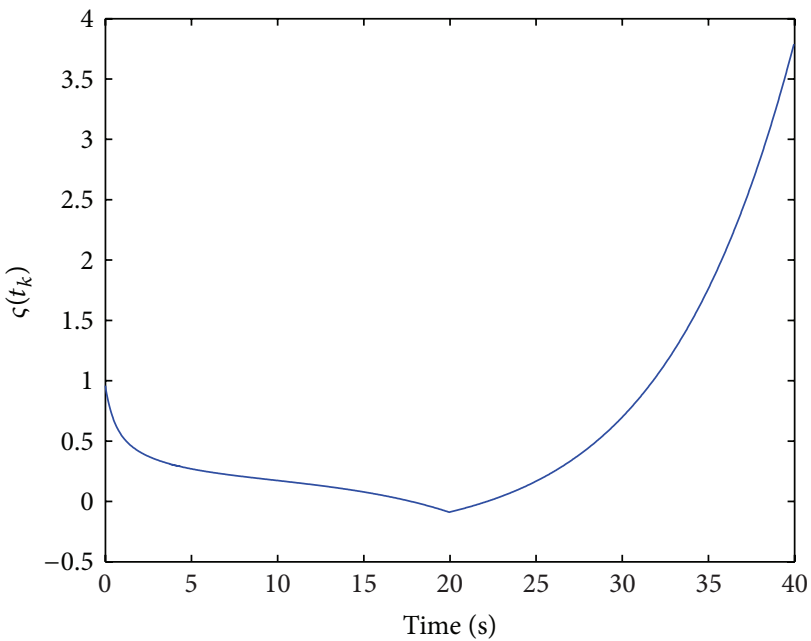

FIgURE 2: Response of $\varsigma\left(t_{k}\right)$ of the open-loop system.

Define the value range of time delay $d_{k}=n T$, and choosing the lower bound $d_{m}=0.01$, the sampling period $T$ may be assumed as $T=0.001$ and it can be found from Theorem 9 that the maximum upper bound of time delay $d_{k}$ and the control gain matrices are listed in Table 1.

Assume that time delay $d_{k}=n T=40 T$ and the disturbance input $w\left(t_{k}\right)=-1 /\left(2+t_{k}\right)$. It can be calculated 


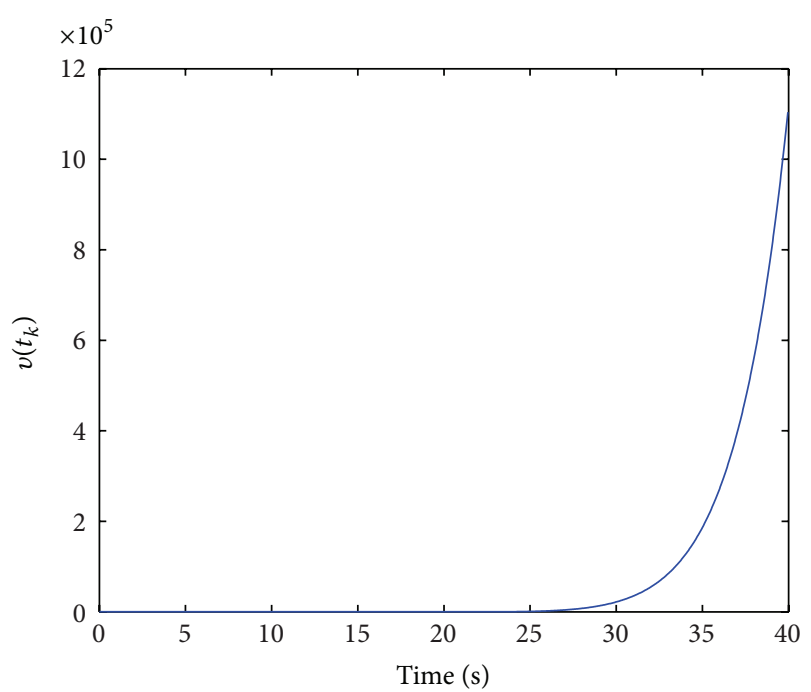

FIgURE 3: Response of $v\left(t_{k}\right)$ of the open-loop system.

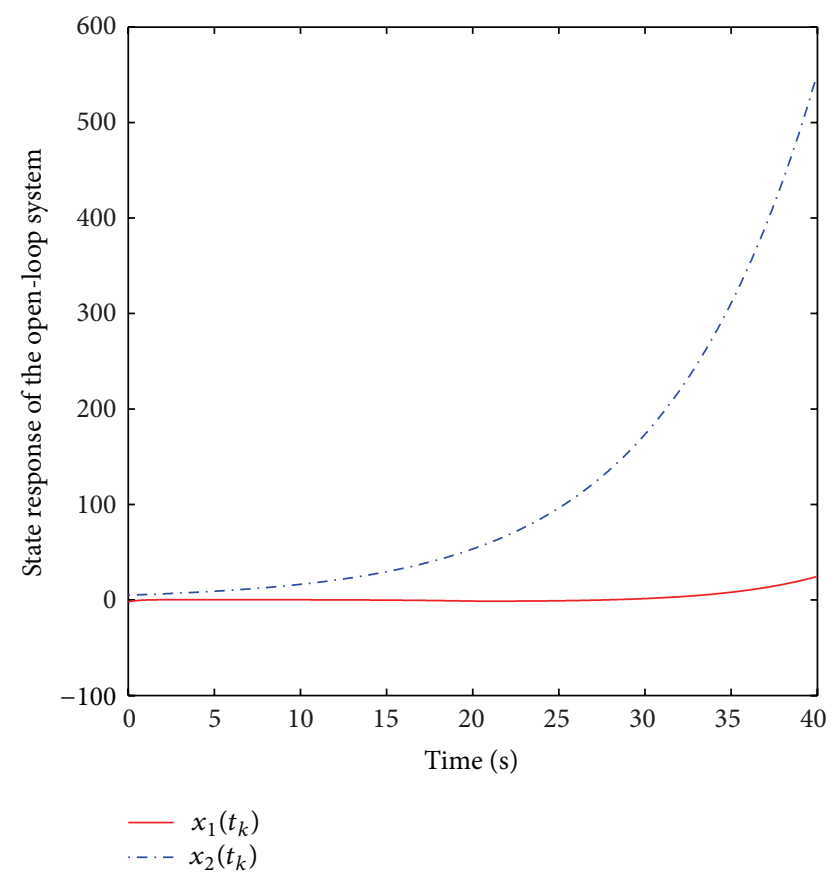

FIGURE 4: State response of the open-loop system.

that $\sum_{t_{k}=0}^{\infty} w^{T}\left(t_{k}\right) w\left(t_{k}\right)=0.5<\infty$, which means that $w\left(t_{k}\right) \in$ $L_{2}[0, \infty)$. Let

$$
\begin{aligned}
& v\left(t_{k}\right)=2 \sum_{t_{k}=0}^{\infty} z^{T}\left(t_{k}\right) w\left(t_{k}\right)-\varepsilon \sum_{t_{k}=0}^{\infty} z^{T}\left(t_{k}\right) z\left(t_{k}\right) \\
& -\zeta \sum_{t_{k}=0}^{\infty} w^{T}\left(t_{k}\right) w\left(t_{k}\right) \\
& \varsigma\left(t_{k}\right)=2 \sum_{t_{k}=0}^{\infty} z^{T}\left(t_{k}\right) w\left(t_{k}\right) \text {. }
\end{aligned}
$$

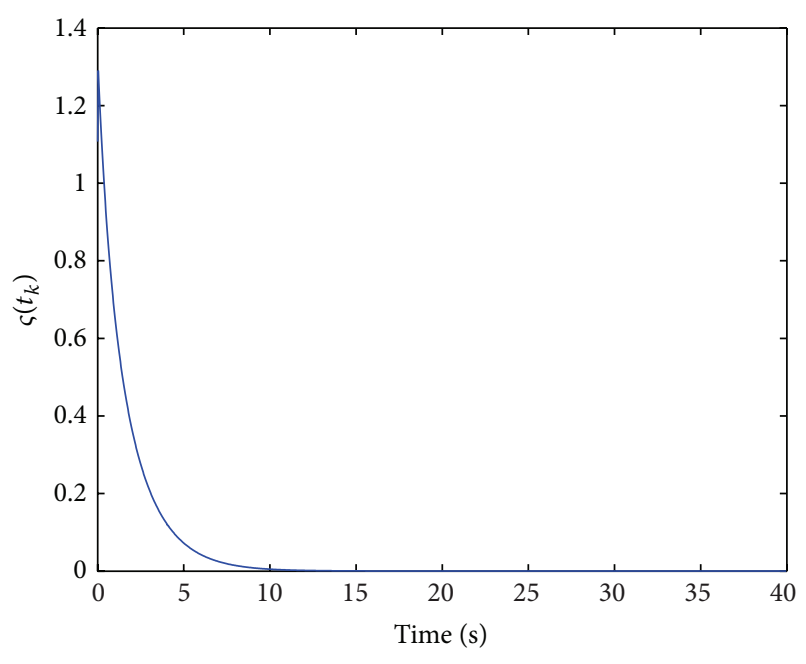

FIGURE 5: Response of $\varsigma\left(t_{k}\right)$ of the closed-loop system.

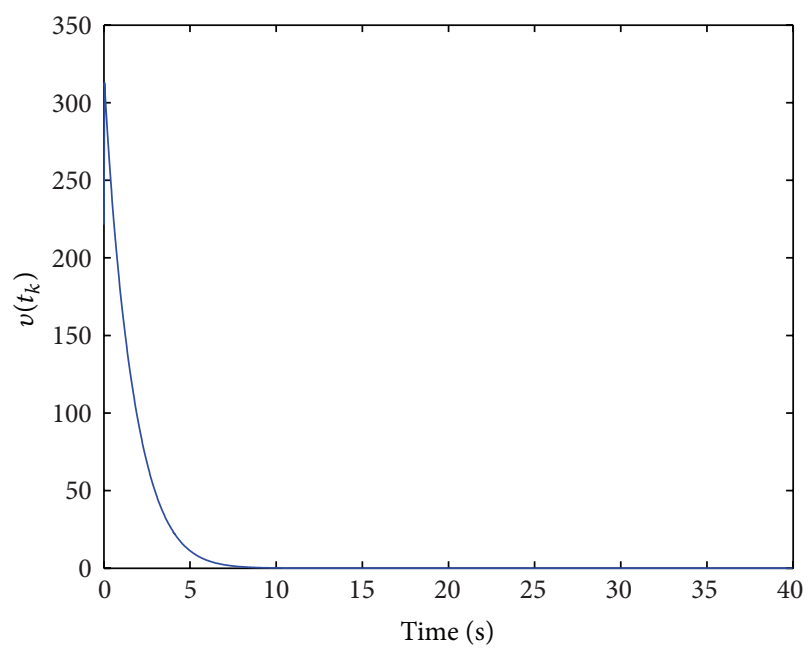

FIGURE 6: Response of $v\left(t_{k}\right)$ of the closed-loop system.

In Figure 2, it can be observed that $\varsigma\left(t_{k}\right)$ decreases finally as the time $t_{k}$ increases, which means that there may not exist a scalar $\rho$ such that $\varsigma\left(t_{k}\right) \geq \rho$ hold for all $t_{k} \geq 0$ for $T=0.001$. Furthermore, Figure 3 shows that there may not exist a scalar $\rho$ such that $v\left(t_{k}\right) \geq \rho$, which means that the open-loop system is not passive in the sense of Definition 2, and it is not very strictly passive. Figure 4 illustrates that the open-loop system is not stable for $T=0.001$.

Under the control gain matrices in Table 1, Figure 5 plots the responses of $\varsigma\left(t_{k}\right)$ for the closed-loop system for $T=$ 0.001 . It can be seen that there may exist a scalar $\rho$ such that $\varsigma\left(t_{k}\right) \geq \rho$ holds for all $t_{k} \geq 0$. It can be seen from Figure 6 that there may exist a scalar $\rho$ such that $v\left(t_{k}\right) \geq \rho$. Then, it is clear that the closed-loop system is very strictly passive under the control gain matrices in Table 1. In addition, Figure 7 shows that the closed-loop system is stable. Furthermore, Figure 8 depicts the control input responses. These simulation results have demonstrated the effectiveness of the proposed method. 


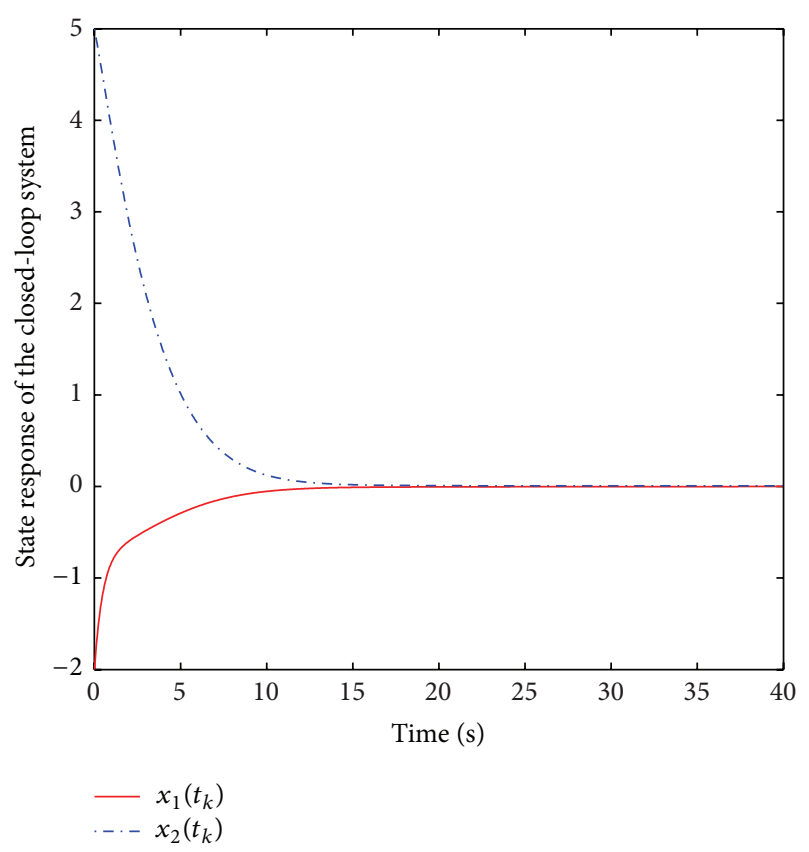

FIGURE 7: State response of the open-loop system.

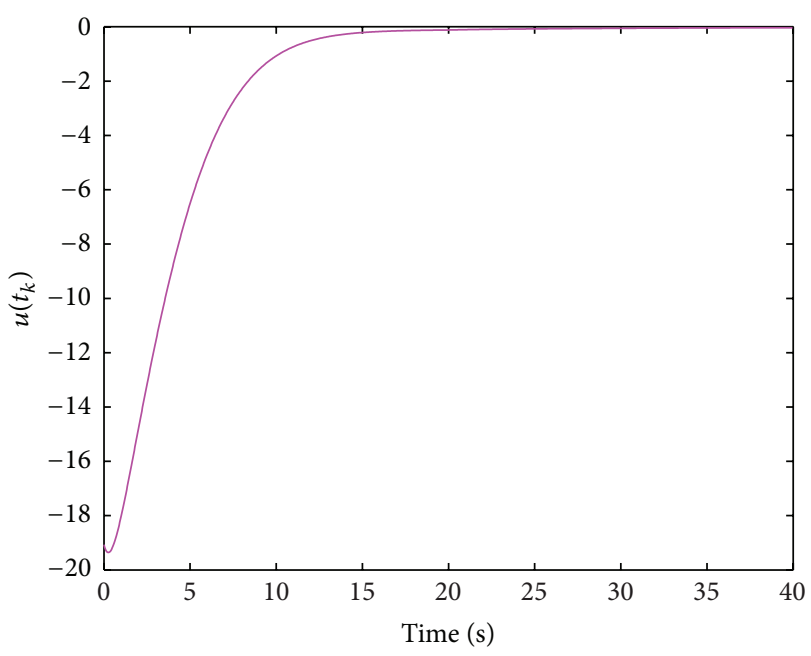

FIGURE 8: Control signal response of the closed-loop system.

\section{Conclusions}

In this paper, the problems of passivity analysis and passive control have been investigated for fuzzy delta operator systems with time delay and disturbance input. By applying some new LKF in $\delta$-domain and utilizing some fuzzy weighing matrices, the state-feedback controller has been designed to guarantee that the resulting closed-loop system is very strictly passive. The existence conditions for the controller have been expressed as LMIs. Finally, a numerical example has been included to illustrate the effectiveness of the proposed results. In future work, based on T-S fuzzy control method, the problems of control and monitoring in the data-driven framework $[53,54]$ could be further studied.

\section{Conflict of Interests}

The authors declare that there is no conflict of interests regarding the publication of this paper.

\section{Acknowledgments}

This work was partially supported by the National Natural Science Foundation of China (61203002, 61304002, and 61304003), the Program for New Century Excellent Talents in University (NCET-13-0696), the Program for Liaoning Innovative Research Team in University (LT2013023), and the Program for Liaoning Excellent Talents in University (LR2013053).

\section{References}

[1] S. Tong, X.-L. He, and H.-G. Zhang, "A combined backstepping and small-gain approach to robust adaptive fuzzy output feedback control," IEEE Transactions on Fuzzy Systems, vol. 17, no. 5, pp. 1059-1069, 2009.

[2] S. Tong and Y. Li, "Observer-based fuzzy adaptive control for strict-feedback nonlinear systems," Fuzzy Sets and Systems, vol. 160, no. 12, pp. 1749-1764, 2009.

[3] Y. Li, S. Tong, Y. Liu, and T. Li, "Adaptive fuzzy robust output feedback control of nonlinear systems with unknown dead zones based on small-gain approach," IEEE Transactions on Fuzzy Systems, 2013.

[4] Y. Li, S. Tong, and T. Li, "Adaptive fuzzy output feedback control of uncertain nonlinear systems with unknown backlash-like hysteresis," Information Sciences, vol. 198, pp. 130-146, 2012.

[5] T. Takagi and M. Sugeno, "Fuzzy identification of systems and its applications to modeling and control," IEEE Transactions on Systems, Man and Cybernetics, vol. 15, no. 1, pp. 116-132, 1985.

[6] K. Tanaka, T. Hori, and H. O. Wang, "A multiple Lyapunov function approach to stabilization of fuzzy control systems," IEEE Transactions on Fuzzy Systems, vol. 11, no. 4, pp. 582-589, 2003.

[7] T. M. Guerra and L. Vermeiren, "LMI-based relaxed nonquadratic stabilization conditions for nonlinear systems in the Takagi-Sugeno's form," Automatica, vol. 40, no. 5, pp. 823-829, 2004.

[8] K. Tanaka and H. O. Wang, Fuzzy Control Systems Design and Analysis. A Linear Matrix Inequality Approach, John Wiley and Sons, New York, NY, USA, 2001.

[9] G. Feng, "A survey on analysis and design of model-based fuzzy control systems," IEEE Transactions on Fuzzy Systems, vol. 14, no. 5, pp. 676-697, 2006.

[10] S. Nguang and P. Shi, " $H_{\infty}$ fuzzy output feedback control design for nonlinear systems: an LMI approach," IEEE Transactions on Fuzzy Systems, vol. 11, no. 3, pp. 331-340, 2003.

[11] S. Nguang, W. Assawinchaichote, and P. Shi, "Robust $H_{\infty}$ control design for fuzzy singularly perturbed systems with Markovian jumps: an LMI approach," IET Control Theory and Applications, vol. 1, no. 4, pp. 893-908, 2007.

[12] B. Jiang, Z. Mao, and P. Shi, " $H_{\infty}$-filter design for a class of networked control systems via TS fuzzy-model approach," IEEE Transactions on Fuzzy Systems, vol. 18, no. 1, pp. 201-208, 2010.

[13] H. Dong, Z. Wang, D. W. C. Ho, and H. Gao, "Robust $H_{\infty}$ fuzzy output-feedback control with multiple probabilistic delays and 
multiple missing measurements," IEEE Transactions on Fuzzy Systems, vol. 18, no. 4, pp. 712-725, 2010.

[14] L. Wu, X. Su, P. Shi, and J. Qiu, "Model approximation for discrete-time state-delay systems in the TS fuzzy framework," IEEE Transactions on Fuzzy Systems, vol. 19, no. 2, pp. 366-378, 2011.

[15] M. Liu, X. Cao, and P. Shi, "Fault estimation and tolerant control for fuzzy stochastic systems," IEEE Transactions on Fuzzy Systems, vol. 21, no. 2, pp. 221-229, 2013.

[16] M. Liu, X. Cao, and P. Shi, "Fuzzy-model-based fault tolerant design for nonlinear stochastic systems against simultaneous sensor and actuator faults," IEEE Transactions on Fuzzy Systems, vol. 21, no. 5, pp. 789-799, 2013.

[17] J. Qiu, G. Feng, and H. Gao, "Fuzzy-model-based piecewise $H_{\infty}$ static-output-feedback controller design for networked nonlinear systems," IEEE Transactions on Fuzzy Systems, vol. 18, no. 5, pp. 919-934, 2010.

[18] J. Qiu, G. Feng, and H. Gao, “Asynchronous output-feedback control of networked nonlinear systems with multiple packet dropouts: T-S fuzzy affine model-based approach," IEEE Transactions on Fuzzy Systems, vol. 19, no. 6, pp. 1014-1030, 2011.

[19] B. Chen, X. Liu, S. Tong, and C. Lin, "Guaranteed cost control of T-S fuzzy systems with state and input delays," Fuzzy Sets and Systems, vol. 158, no. 20, pp. 2251-2267, 2007.

[20] B. Chen, X. Liu, C. Lin, and K. Liu, "Robust $H_{\infty}$ control of Takagi-Sugeno fuzzy systems with state and input time delays," Fuzzy Sets and Systems, vol. 160, no. 4, pp. 403-422, 2009.

[21] S. Xu and J. Lam, "Robust $H_{\infty}$ control for uncertain discretetime-delay fuzzy systems via output feedback controllers," IEEE Transactions on Fuzzy Systems, vol. 13, no. 1, pp. 82-93, 2005.

[22] C. Lin, G. Wang, and T. Lee, LMI Approach to Analysis and Control of Takagi-Sugeno Fuzzy Systems with Time Delay, Springer, New York, NY, USA, 2007.

[23] C. Lin, Q.-G. Wang, and T. H. Lee, "Stabilization of uncertain fuzzy time-delay systems via variable structure control approach," IEEE Transactions on Fuzzy Systems, vol. 13, no. 6, pp. 787-798, 2005.

[24] B. Zhang and S. Xu, "Delay-Dependent Robust $H_{\infty}$ control for uncertain discrete-time fuzzy systems with time-varying delays," IEEE Transactions on Fuzzy Systems, vol. 17, no. 4, pp. 809-823, 2009.

[25] B. Zhang, J. Lam, S. Xu, and Z. Shu, "Robust stabilization of uncertain T-S fuzzy time-delay systems with exponential estimates," Fuzzy Sets and Systems, vol. 160, no. 12, pp. 1720-1737, 2009.

[26] L. Wu, X. Su, P. Shi, and J. Qiu, "A new approach to stability analysis and stabilization of discrete-time T-S fuzzy timevarying delay systems," IEEE Transactions on Systems, Man, and Cybernetics B, vol. 41, no. 1, pp. 273-286, 2011.

[27] H. Li, J. Yu, C. Hilton, and H. Liu, "Adaptive sliding mode control for nonlinear active suspension vehicle systems using TS fuzzy approach," IEEE Transactions on Industrial Electronics, vol. 60, no. 8, pp. 3328-3338, 2013.

[28] H. Li, H. Liu, H. Gao, and P. Shi, "Reliable fuzzy control for active suspension systems with actuator delay and fault," IEEE Transactions on Fuzzy Systems, vol. 20, no. 2, pp. 342-357, 2012.

[29] H. K. Lam and J. Lauber, "Membership-function-dependent stability analysis of fuzzy-model-based control systems using fuzzy Lyapunov functions," Information Sciences, vol. 232, no. 14, pp. 253-266, 2013.
[30] H. K. Lam and J. C. Lo, "Output regulation of polynomial-fuzzymodel-based control systems," IEEE Transactions on Fuzzy Systems, vol. 21, no. 2, pp. 262-274, 2013.

[31] H. K. Lam, "Polynomial fuzzy-model-based control systems: stability analysis via piecewise-linear membership functions," IEEE Transactions on Fuzzy Systems, vol. 19, no. 3, pp. 588-593, 2011.

[32] X. Zhao, L. Zhang, P. Shi, and H. R. Karimi, "Novel stability criteria for T-S fuzzy systems," IEEE Transactions on Fuzzy Systems, 2013.

[33] L. Wu and W. X. Zheng, "Passivity-based sliding mode control of uncertain singular time-delay systems," Automatica, vol. 45, no. 9, pp. 2120-2127, 2009.

[34] H. Gao, T. Chen, and T. Chai, "Passivity and passification for networked control systems," SIAM Journal on Control and Optimization, vol. 46, no. 4, pp. 1299-1322, 2007.

[35] S. Xu, W. X. Zheng, and Y. Zou, "Passivity analysis of neural networks with time-varying delays," IEEE Transactions on Circuits and Systems II, vol. 56, no. 4, pp. 325-329, 2009.

[36] J. Liang, Z. Wang, and X. Liu, "On passivity and passification of stochastic fuzzy systems with delays: the discrete-time case," IEEE Transactions on Systems, Man, and Cybernetics B, vol. 40, no. 3, pp. 964-969, 2010.

[37] C. Wang and Y. Shen, "Passive control for stochastic interval systems with interval time-varying delay," Asian Journal of Control, vol. 15, no. 1, pp. 248-259, 2013.

[38] H. Li, H. Gao, and P. Shi, "New passivity analysis for neural networks with discrete and distributed delays," IEEE Transactions on Neural Networks, vol. 21, no. 11, pp. 1842-1847, 2010.

[39] Z.-G. Wu, P. Shi, H. Su, and J. Chu, "Passivity analysis for discrete-time stochastic markovian jump neural networks with mixed time delays," IEEE Transactions on Neural Networks, vol. 22, no. 10, pp. 1566-1575, 2011.

[40] C. Li, H. Zhang, and X. Liao, "Passivity and passification of fuzzy systems with time delays," Computers and Mathematics with Applications, vol. 52, no. 6-7, pp. 1067-1078, 2006.

[41] B. Zhang, W.X. Zheng, and S. Xu, "Passivity analysis and passive control of fuzzy systems with time-varying delays," Fuzzy Sets and Systems, vol. 174, no. 1, pp. 83-98, 2011.

[42] G. Goodwin, R. L. Leal, D. Q. Mayne, and R. H. Middleton, "Rapprochement between continuous and discrete model reference adaptive control," Automatica, vol. 22, no. 2, pp. 199-207, 1986.

[43] R. Middleton and G. Goodwin, "Improved finite word length characteristics in digital control using delta operators," IEEE Transactions on Automatic Control, vol. 31, no. 11, pp. 1015-1021, 1986.

[44] C. P. Neuman, "Transformation between delta and forward shift operator transfer function models," IEEE Transactions on Systems, Man and Cybernetics, vol. 23, no. 1, pp. 295-296, 1993.

[45] K. Premaratne, R. Salvi, N. R. Habib, and J. P. LeGall, "Delta-operator formulated discrete-time approximations of continuous-time systems," IEEE Transactions on Automatic Control, vol. 39, no. 3, pp. 581-585, 1994.

[46] K. Premaratne and E. Jury, "Tabular method for determining root distribution of delta-operator formulated real polynomials," IEEE Transactions on Automatic Control, vol. 39, no. 2, pp. 352-355, 1994.

[47] M. Tadjine, M. M'Saad, and L. Dugard, "Discrete-time compensators with loop transfer recovery," IEEE Transactions on Automatic Control, vol. 39, no. 6, pp. 1259-1262, 1994. 
[48] J. Qiu, Y. Xia, H. Yang, and J. Zhang, "Robust stabilisation for a class of discrete-time systems with time-varying delays via delta operators," IET Control Theory and Applications, vol. 2, no. 1, pp. 87-93, 2008.

[49] H. Yang, Y. Xia, and P. Shi, "Observer-based sliding mode control for a class of discrete systems via delta operator approach," Journal of the Franklin Institute, vol. 347, no. 7, pp. 1199-1213, 2010.

[50] H. Yang, P. Shi, J. Zhang, and J. Qiu, "Robust $H_{\infty}$ control for a class of discrete time fuzzy systems via delta operator approach," Information Sciences, vol. 184, no. 1, pp. 230-245, 2012.

[51] B. Brogliato, O. Egeland, R. Lozano, and B. Maschke, Dissipative Systems Analysis and Control: Theory and Applications, Springer, London, UK, 2007.

[52] X. Jiang, Q.-L. Han, and X. Yu, "Stability criteria for linear discrete-time systems with interval-like time-varying delay," in 2005 American Control Conference, ACC, pp. 2817-2822, usa, June 2005.

[53] S. Yin, S. Ding, and H. Luo, "Real-time implementation of fault tolerant control system with performance optimization," IEEE Transactions on Industrial Electronics, vol. 61, no. 5, pp. 24022411, 2014.

[54] L. Xie, "Output feedback $H_{\infty}$ control of systems with parameter uncertainty," International Journal of Control, vol. 63, no. 4, pp. 741-750, 1996. 


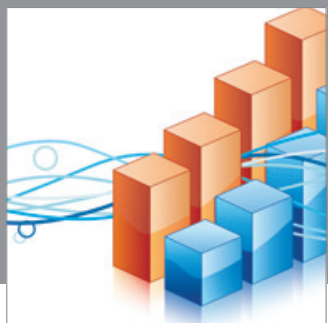

Advances in

Operations Research

mansans

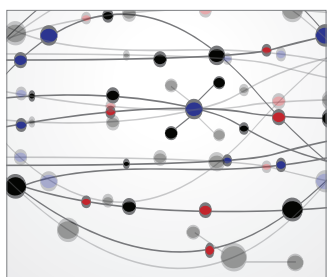

The Scientific World Journal
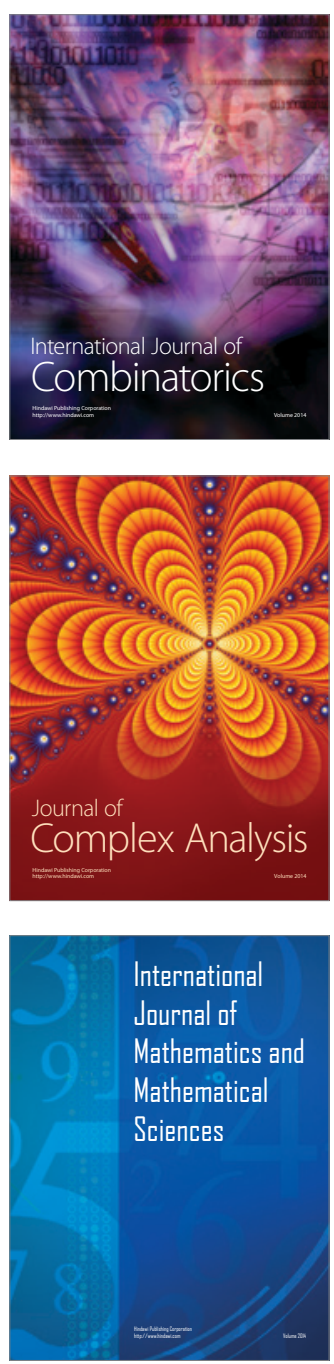
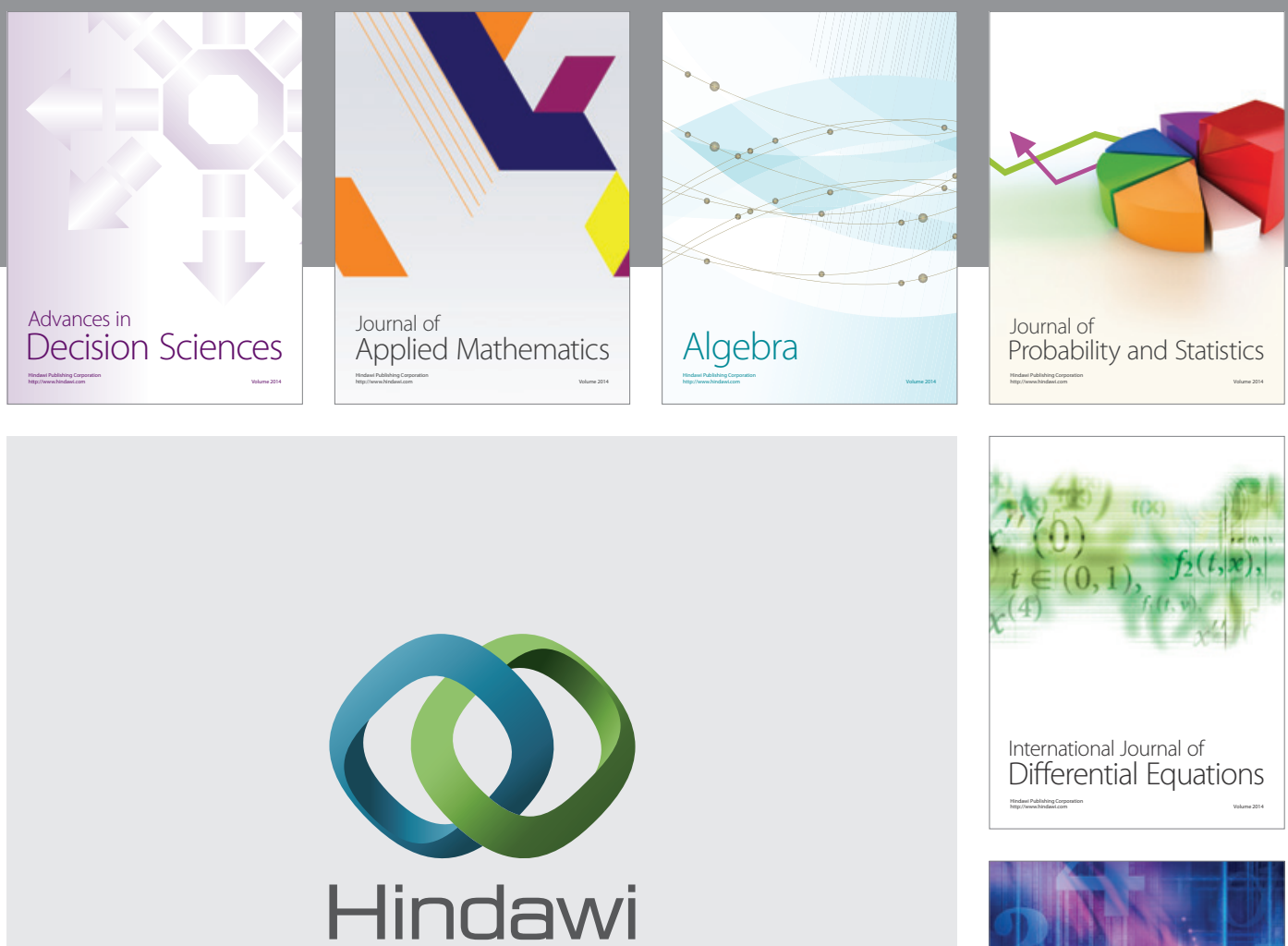

Submit your manuscripts at http://www.hindawi.com
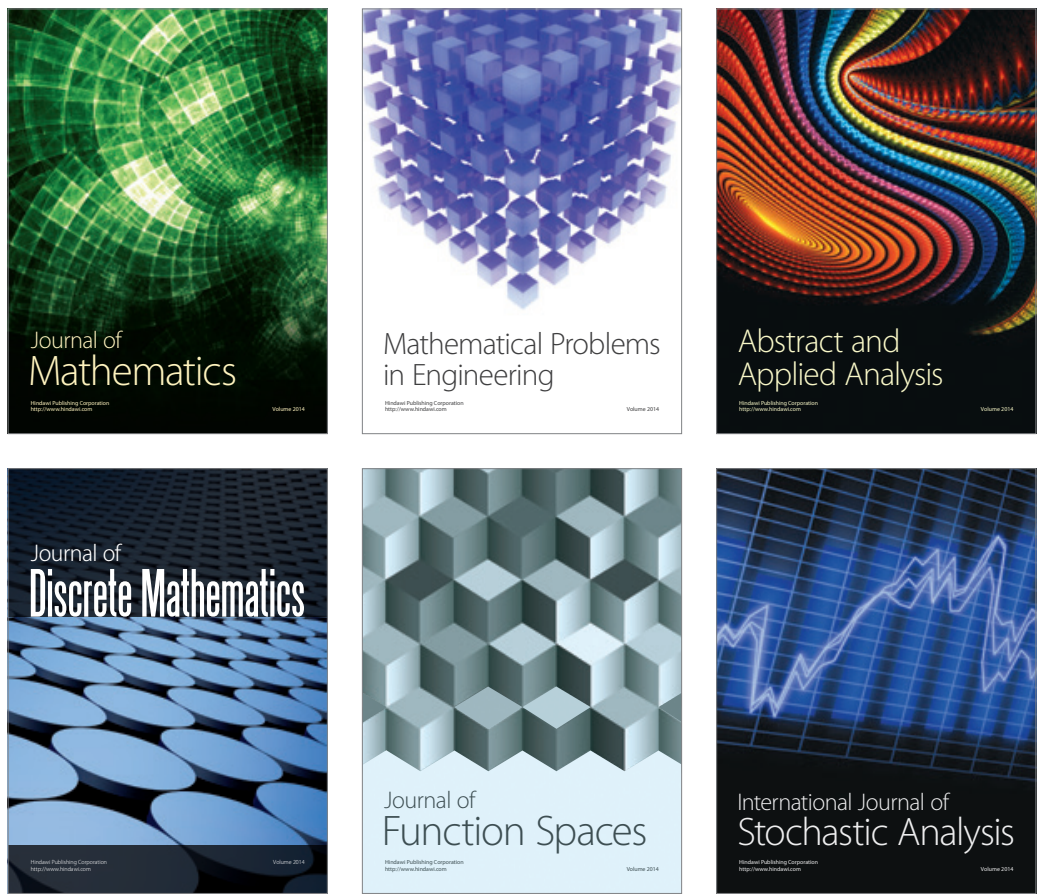

Journal of

Function Spaces

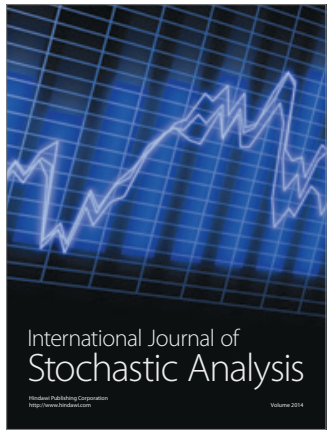

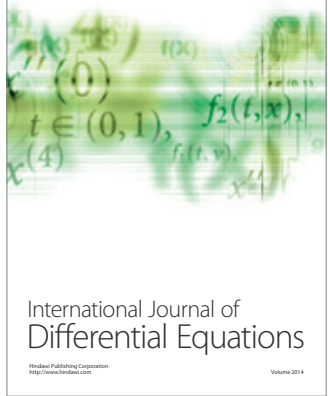
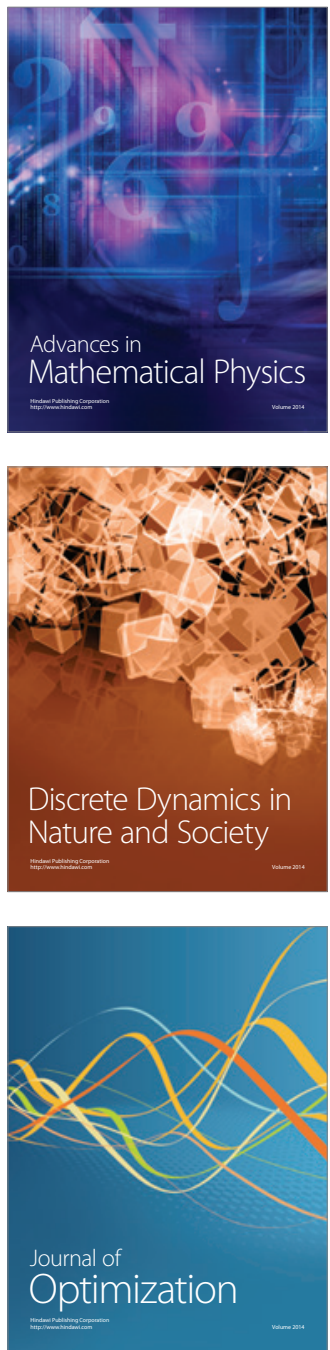